\title{
Cardiovascular Disease Classification using Photoplethysmography Signals- Survey
}

\author{
R. Divya \\ Assistant Professor \\ Department of Electrical and Electronics \\ Engineering \\ PSG College of Technology Coimbatore, India
}

\author{
P. T. Vanathi \\ Associate Professor \\ Department of Electronics and Communication \\ PSG College of Technology Coimbatore, India
}

\begin{abstract}
In recent years, heart disease has been one of the main causes of death worldwide. Statistics report that around 17 million people die every year due to this disease. Usually the heart disease diagnosis is done using Electrocardiogram (ECG) which is very expensive for the people to afford especially in remote areas. But using Photoplethysmography (PPG) signals it is easier, non-invasive and less expensive in detecting the heart diseases and other abnormalities of human body. So in this paper, various PPG signals usage and their merits are discussed. Also this work focuses on several methods and algorithms of cardiovascular disease (CVD) classification. Several classifier techniques in the field of biomedical signal processing methods are also examined.
\end{abstract}

\section{Keywords}

Cardiovascular disease, Heart rate, Peak-to-peak interval Photoplethysmography.

\section{INTRODUCTION}

Biomedical signals are observations of physiological activities of organisms, ranging from gene and protein sequences, to neural and cardiac rhythms, to tissue and organ images. With the aid of biomedical signal processing, here a survey is examined that finds various cardio vascular diseases and other heart related diseases. So that biologists can discover new biology and physicians can monitor distinct illnesses.

Cardiovascular diseases (CVD), which include diseases of the heart, are the leading cause of death worldwide. It is a very serious health condition that keeps the heart or blood vessels from working properly $[1,2]$. There are many cardiovascular diseases involving the blood vessels. It is also estimated that consistently, more than 1 million individuals in the United States and > 19 million others overall experience a sudden heart problems [3]. Diseases affecting the cardiovascular system are called cardiovascular disease. There are many risk factors for heart diseases: age, gender, tobacco use, physical inactivity, excessive alcohol consumption, unhealthy diet, obesity, genetic predisposition and family history of cardiovascular disease, raised blood pressure (hypertension), raised blood sugar (diabetes mellitus), raised blood cholesterol (hyperlipidemia), psychosocial factors, poverty and low educational status, and air pollution [4]. Many of these diseases are called "lifestyle diseases" because they develop over time and are related to a person's exercise habits, diet, whether they smoke, and other lifestyle choices a person makes [5]. Cardiovascular disease is a class of diseases that involve the heart or blood vessels. Cardiovascular disease includes coronary artery disease such as angina and myocardial infarction (commonly known as a heart attack). Other CVDs include stroke, heart failure, hypertensive heart disease, rheumatic heart disease, cardiomyopathy, heart arrhythmia, congenital heart disease, valvular heart disease, carditis, aortic aneurysms, peripheral artery disease, thromboembolic disease, and venous thrombosis.

The CVD diagnosis methods are discussed as follows. Electrocardiogram (ECG or EKG) records the electrical activity of the heart as it contracts and relaxes. The ECG can detect abnormal heartbeats, some areas of damage, inadequate blood flow, and heart enlargement. Next, blood test method checks for enzymes or other substances released when cells begin to die. They are "markers" of the amount of damage to your heart. Then, nuclear scan test reveals the damaged areas of the heart that lack blood flow. It also can show problems with the heart's pumping action. The test uses radioactive tracers to study how blood flows in your heart. Coronary angiography is another test that uses dye and special $\mathrm{X}$-rays to show the inside of your coronary arteries.It is estimated that $90 \%$ of CVD is preventable. To address the socioeconomic differentials in the burden of disease and healthcare needs, more resources need to be directed toward applying the existing knowledge base to tackle the CVD epidemic in policy, programs, capacity building, and research areas.

\section{PHOTOPLETHYSMOGRAPHY}

A photo plethysmogram (PPG) is an optically obtained plethysmogram, a volumetric measurement of an organ. A PPG is often obtained by using a pulse oximeter which illuminates the skin and measures changes in light absorption. The change in volume caused by the pressure pulse is detected by illuminating the skin with the light from a light-emitting diode (LED) and then measuring the amount of light either transmitted or reflected to a photodiode. A pulse oximeter estimates the amount of oxygen carried in the bloodstream using infrared technology across the skin. It gives the blood oxygen level (SpO2) and heart rate [6].

Plethysmographs measure changes in volume. The volume change can be converted into blood flow by using $\mathrm{F}=\mathrm{dV} / \mathrm{dt}$. In some cases, only relative volume needs to be been known, for example to see the pulsation rate of the heart. In that case, information is in the timing and not in the amplitude or shape of the signal. If that is the case, then electric impedanceplethysmography or photo-plethysmography (PPG) can be used. Thus PPG is a simple and low-cost optical technique that can be used to detect blood volume changes in the microvascular bed of tissue. It is often used non-invasively to make measurements at the skin surface.

\section{LITERATURE SURVEY}

Some of the literature work about the PPG analysis and the heart disease classification methods are listed in the following. The main literature work is to determine whether the photoplethysmography can replace the ECG-based detection of heart rate turbulence. Fig.1 represents the common structure for diagnostic system of PPG signal. 


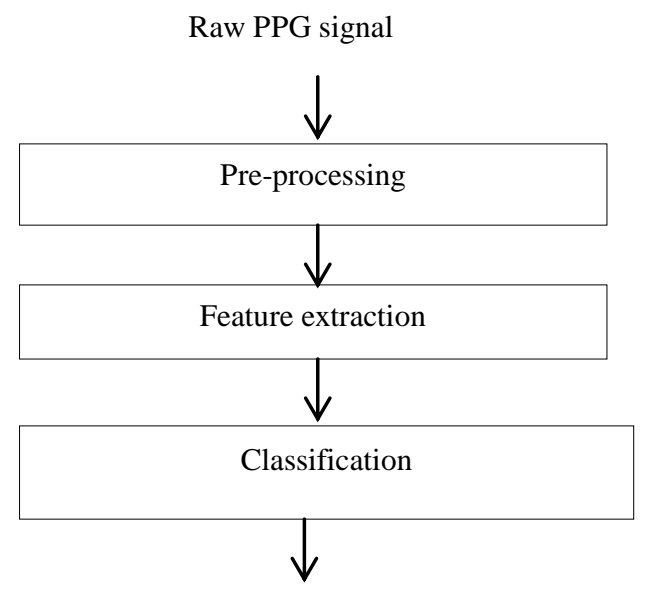

Disease Diagnosis

\section{Figure 1 Diagnostic system of PPG signal}

As shown in Figure 1, diagnostic system of PPG signal consists of pre-processing stage, feature extraction stage and classification stage.

There are many factors which may generate artifacts within the PPG signals. Powerline interference due to instrumentation amplifier, poor contact to the photosensor, sudden changes in the amplitude due to the automatic gain controller, premature ventricular contraction affecting the main events detection accuracy in PPG signals are some of the causes for the artifacts [7]. So these artifacts are to be removed in the pre-processing stage. Feature extraction is a dimensionality reduction process, where an initial set of raw variables is reduced to more manageable features for processing, while still accurately and completely describing the original data set. Features extracted from the PPG signals are given as input to the classifier for disease diagnosis.By using the PPG, classification of ventricular premature beats (VPBs) is accomplished with a linear classifier by Gil et al. (2013) [8]. Performance is studied on a dataset with 4131 VPBs, recorded from a total of 27 patients in different clinical contexts (hemodialysis treatment, intensive care monitoring, and electrophysiological study). The sensitivity/specificity of VPB classification was found to be $90.5 / 99.9 \%$, with an accuracy of $99.3 \%$, suggesting that classification of VPBs can be reliable made from the PPG. Out of the 22 patients who had a sufficient number of VPBs, the outcome of the ECG and PPG-based analysis was identical in 21 . It is concluded that the PPG may serve as a surrogate technique for the ECG in turbulence analysis.

In particular, photoplethysmography is especially suitable for wearable sensing, as it is low-cost, noninvasive, and does not require wet electrodes like the electrocardiogram. Photoplethysmograph signals contain rich information about the blood pulsating variation which is strongly related to the electrical activities of the heart. Therefore, Polania et al. (2015) hypothesized that the ambulatory PPG monitoring could be employed for arrhythmia detection and classification. Also it presents a method for classifying ventricular premature contraction (VPC) and ventricular tachycardia (VT) from normal sinus rhythm (NSR) and supraventricular premature contraction (SVPC) recorded in patients going through ablation therapy for arrhythmia.
Karlen et al. (2013) introduced a novel technique for evaluating respiratory rate progressively from the photoplethysmogram acquired from beat oximetry [10]. Three respiratory-actuated varieties (recurrence, power, and sufficiency) are removed from the PPG utilizing the Incremental-Merge Segmentation calculation. Recurrence substance of each respiratory-prompted variety is broke down utilizing quick Fourier changes. The proposed smart fusion strategy at that point joins the results of the three respiratoryactuated varieties utilizing a straightforward mean estimation. It naturally wipes out estimations thought to be temperamental in view of identified nearness of ancient rarities in the PPG or contradiction between the diverse individual respiratory rate estimations. The calculation has been tried on information acquired from 29 youngsters and 13 grown-ups. Results demonstrate that it is imperative to consolidate the three respiratory-prompted varieties for powerful estimation of respiratory rate. The smart fusion demonstrated patterns of enhanced estimation (mean root mean square mistake 3.0 breaths/min) contrasted with the individual estimation strategies $(5.8,6.2$, and 3.9 breaths $/ \mathrm{min})$. The smart fusion calculation is being actualized in a cell phone beat oximeter gadget to encourage the finding of serious youth pneumonia in remote territories.

Heart rate checking has turned out to be progressively well known in the industry through cell phones and wearable gadgets. Nonetheless, current assurance of heart rate through mobile applications experiences high corruption of signals during intensive physical exercise. Vasu Jindal (2016) proposed a novel strategy for precisely determining the heart rate during intensive motion by classifying the PPG signals got from cell phones or wearable gadgets joined with motion information acquired from accelerometer sensors is exhibited. Their approach uses the Internet of Things (IoT) cloud availability of cell phones for determination of PPG signals utilizing profound learning. The procedure is approved utilizing the TROIKA dataset and is precisely ready to anticipate heart rate with a 10 -fold cross validation error margin of $4.88 \%$.

Asif et al. (2011) developed a simple and effective method of displaying blood pressure measurement from photoplethysmography [12]. The method is developed to be incorporated as noninvasive monitoring of blood pressure from a patient in an intensive care unit. This implementation includes fingertip pulse oximetry probe, differential amplifier, data acquisition card, software filtering, amplification and blood pressure measurement from pulse transit time calculation continuously by microcomputer correlation of pulse transit time to blood pressure. It is expected that the method will be incorporated to a low cost portable personal computer based ICU monitoring system that will also display temperature, pulse rate and ECG of the patient continuously in future.

Photoplethysmography signals are always corrupted with motion artifacts and resulting in bad quality PPG signals. The proper designed data acquisition tool may help in acquisition of reliable and consistence PPG signal. However, in most cases, the acquired PPG are bad quality signals. The repeated acquisition sessions sometimes cannot be performed due to ethical issues and time consuming. As such an essential step is needed to deal with the corrupted PPG signal. So, Nurhafizah Mahri (2012) proposed an algorithm to select best pulse in PPG signal through comparative method of valley detection. The clinical data showed that this algorithm is a promising 
tool to analyze low quality PPG signals especially during data acquisition.

Yousef et al. (2012) presented the varieties of photoplethysmogram morphology with age is contemplated [14]. PPG estimation is done noninvasively at the pointer on both right and left hands for an example of erectile dysfunction (ED) subjects. A few parameters are obtained from the investigation of PPG signal appeared in relationship with age. The age is observed to be an essential factor that influences the contour of PPG signal which quickens the vanishing of PPG's dicrotic notch and PPG's inflection point. Blood vessel consistence is observed to be corrupted with age because of the fall of blood vessel versatility. This examination approaches the foundation of usefulness of PPG's contour analysis as an examiner to the adjustments in the versatile properties of the vascular system, and as an identifier of early sub-clinical atherosclerosis.

The design and implementation of a microcontroller-based embedded system for blood pressure monitoring through a Photoplethysmographic technique is presented by Agro et al. (2014). In this system, it is possible to perform PPG measurements via reflectance mode. Hardware novelty of the system consists in the adoption of Silicon Photomultiplier detectors. The signal received from the photo detector is used to calculate the instantaneous heart rate and therefore the heart rate variability [15]. The obtained results show that, it is possible to easily extract both the PPG and the breath signal. These signals can be used to monitor the patients during the convalescence both in hospital and at home.

Miguel Tobal et al. (2010) reported the utilization of transmittance PPG all through maximal exercise tests in cycle ergometer in male athletes [16]. A specially developed algorithm has been applied to get PPG beat rates like the reference heart rates. The point of this examination has been to survey that heartbeat rate from photoplethysmographic signals, even in maximal exercise tests in cycle ergometer, is a true estimate of the heart rate or cardiac frequency acquired by electrocardiography. In states of serious exercise, the harmonic content of the signal could be very complex because of the strong movement artifacts. The different frequencies got from the movement artifacts and pulse, and in addition their amplitude distribution, make essential usage of an algorithm that can separate, among every one of the frequencies, one comparing to the heart beat. Specially developed algorithm has been applied to the PPG signs of one emitter close to $850 \mathrm{~nm}$, which were recorded utilizing a fingertip sensor in 18 athletes. The relative examination of our PPG derived pulse rates demonstrates the agreement with heart rates by ECG, even for high rates, with small errors and the greater part of the values within the error bands, in both independent groups of tested athletes. This demonstrates the possibility of photoplethysmography for beat rate evaluation all through intensive exercise in cycle ergometer.

The physiological parameters that are utilized as risk factors for the prediction of cardiovascular events, and outlining the present status on the medicinal devices for physiological tests has been investigated by Lin et al. (2013). The outcomes demonstrate that measures extracted from blood pressure, electrocardiogram, arterial stiffness, and ankle-brachial blood pressure index (ABI), and blood glucose carry useful information for the forecast of both long term and near-term cardiovascular risk. However, the predictive values should be further validated by more comprehensive measures. In the meantime, the predictive values should be further validated by more comprehensive measures [17]. In the meantime, advancing unobtrusive technologies and wireless communication technologies allow on-site detection of the physiological information remotely in an out-of-hospital setting in real-time. It may allow for personalized, quantitative, and real-time assessment of sudden CVD events.

The classes of heart disease utilizing support vector machine (SVM) as well as artificial neural network (ANN) are incorporated by Rhadhimeenakshi (2016). Examination is completed among two strategies on the premise of accuracy and training time. This paper introduces a medical choice backing framework for coronary illness characterization in a sensible, purpose, precise and fast manner. The dataset utilized are the Cleveland Heart Database and Statlog Database taken from UCI Machine learning dataset vault. In the proposed system model the data records is arranged into two classes in Support Vector Machine as well as Artificial Neural Network. The outputs from SVM and ANN are compared and the differences are noted. From which, the SVM classification is enhanced by keeping away from repetitive searches with a specific end goal to locate the best points to use in predicting. Also the accuracy is found to be high in SVM. In case of ANN, the speed or velocity can be accelerated to enhance the accuracy performance [18].

In the study, Selvaraj et al. (2008) recorded finger-tip PPG and standard lead II ECG for five minutes from 10 healthy subjects at rest. The results showed a high correlation (median $=0.97$ ) between the ECG-derived RR intervals and PPGderived peak-to-peak (PP) intervals. PP variability was accurate $(0.1 \mathrm{~ms})$ as compared to RR variability. The time domain, frequency domain and Poincare plot HRV parameters computed using RR interval method and PP interval method showed no significant differences $(p<0.05)$. The error analysis also showed insignificant differences between the HRV indices obtained by the two methods [19]. BlandAltman analysis showed high degree of agreement between the two methods for all the parameters of HRV. Thus, HRV can also be reliably estimated from the PPG based PP interval method.

Allen (2007) briefed the photoplethysmography and its application in clinical physiological measurement [20]. The PPG technology has been used in clinical physiological estimations, including clinical physiological checking, vascular evaluation and assessing autonomic functions. In spite of the fact that the sources of the parts of the PPG signal are not completely comprehended, it is by and large acknowledged that they can give significant data about the cardiovascular system. There has been a resurgence of interest in the technique for the system, determined by the interest for minimal effort, basic and compact innovation for the essential care and community based clinical settings, the wide accessibility of ease and little semiconductor parts, and the progression of computer-based heartbeat wave examination strategies.

The principle of photoplethysmography, optical techniques and instrumentation frameworks, PC based heartbeat wave examination, and for each situation identified with the clinical use of vascular infection identification is briefed by John Allen et al. (2014). A clinical evaluation of a patient with suspected vascular disorder would include taking a therapeutic history and carrying out an exhaustive physical examination. The medicinal history would help to set up risk factors for vascular disease. The physical examination would include the meticulous palpation of the leg pulses to check whether they were debilitated or absent [21]. Ideally, an underlying vascular screening evaluation is required, which 
precisely decides whether a patient has normal arteries or if they should be referred to an expert for an exhaustive cardiovascular assessment.

Photoplethysmogram signals of 30 normal and 30 pathological, are named healthy or in risk of cardiovascular disease (CVDs) utilizing extremely learning machine (ELM), a supervised learning algorithm is presented by Shobitha et al. (2016). Moreover, two other supervised learning algorithms, back propagation and support vector machine (SVM) are utilized for classifications to compare their results with that of ELM and subsequently its performance has been validated. From the results, it is inferred that ELM gives the best accuracy, a sensitivity of $89.33 \%$ and a specificity of $90.33 \%$, with least training time and least number of features as input [22].For affordable cardiac health monitoring, it is required to ensure accurate cardiac condition detection from smartphone or wearable-extracted photoplethysmogram signals through precise identification, and removal of signal corruption as mentioned in [23]. Presence of noise particularly due to motion artifacts strongly impacts the outcome of analysis. Denoising of PPG signal is established here for better clinical prediction than analyzing the signal in presence of noise. It is proved that analyzing on cleaned (denoised) PPG signal yields significant performance efficacy improvement while performing Coronary Artery Disease (CAD) identification. The proposed method is independent on the clinical analytics and CAD detection is considered to be a use case to justify that physiological signal pre-processing, specifically denoising can substantially improve the overall performance effectiveness and clinical utility.

Sunil and Harikumar (2015) analyzed the PPG signal with respect to the parameters like Principal Component Analysis (PCA), Independent Component Analysis (ICA), Mutual Information (MI) and Entropy. PPG has additionally ended up being a standout amongst the most promising technologies for the early screening of heart related pathologies [23]. Likewise, PPG signals are examined concerning the parameters like Expectation Maximization (EM), Minimum Expectation Maximization (MEM) and Center Tendency Moment (CTM).

A method for detection of premature ventricular contractions (PVCs) in PPG was introduced by Solosenko et al. (2015). The method relies on 6 features, characterizing PPG pulse power, and peak-to-peak intervals. A sliding window approach is applied to extract the features, which are later normalized with respect to an estimated heart rate. Artificial neural network with either linear or non-linear outputs was investigated as a feature classifier [25]. PhysioNet databases, namely, the MIMIC II and the MIMIC, were used for training and testing, respectively. After annotating the PPGs with respect to synchronously recorded electrocardiogram, two main types of PVCs were distinguished: with and without the observable PPG pulse. The obtained sensitivity and specificity values for both considered PVC types were 92.4 / 99.9\% and $93.2 / 99.9 \%$, respectively. The achieved high classification results form a basis for a reliable PVC detection using a less obtrusive approach than the electrocardiography-based detection methods.

PPG signals can be utilized as a part of clinical evaluation, for example, heart rate estimations and extraction of blood vessel stream waveforms as in [26]. Motion artifacts and poor peripheral perfusion can contaminate the PPG during observing. A computational framework is exhibited here to limit these two intrinsic shortcomings of the PPG signals. In particular, accelerometers are utilized to recognize the presence of motion artifacts and an adaptive filter is utilized to

minimize the induced errors. Zero-phase digital filtering is engaged to reduce inaccuracy on the PPG signals when estimated from a poorly perfused periphery. In this system, a decision matrix adopts the proper strategy to enhance the PPG technique to improve signal-to-noise ratio dynamically. Measurable examinations demonstrate promising outcomes (maximum error $<7.63 \%$ ) when computed heart rate is contrasted with comparing estimates from the electrocardiogram. Henceforth, the results show that this double mode approach has potential for use in applicable clinical estimations.

Sangita Das et al. (2016) proposed PPG based noise robust real time heart rate measurement technique [27]. It has been developed using Arduino Uno board based on 8-bit AVR core microcontroller and having $16 \mathrm{MHz}$ clock frequency. The basic idea of the work is to extract the periodic PPG signal contaminated by non-periodic noise and artifact. The algorithm is based on short term autocorrelation technique over the time shifted PPG signal. The algorithm and the developed system are validated against the heart rates derived from the signals acquired in BIOPAC MP150 data acquisition system. The designed system is highly noise robust and it can detect heart rate with almost $0 \%$ error considering BIOPAC MP150 as a standard.

The classification accuracy of the PPG signal depends on the appropriate feature selection and classifiers. Table 1 shows the summary of previous works for the cardiovascular assessment from the PPG signals.

Table 1. Summary of previous works for the cardiovascular assessment from the PPG signals

\begin{tabular}{cccc}
\hline Ref. & $\begin{array}{c}\text { Data } \\
\text { set }\end{array}$ & $\begin{array}{c}\text { Feature } \\
\text { extraction }\end{array}$ & Classification \\
\hline$[8]$ & 27 & $\begin{array}{c}\text { Pulse rate } \\
\text { turbulence, } \\
\text { pulse transit } \\
\text { time, }\end{array}$ & $\begin{array}{c}\text { Linear classifier-Ventricular } \\
\text { premature beats (VPBs) or } \\
\text { normal with sensitivity of } \\
90.5 \% \text {, specificity of } 99.9 \% \text { and } \\
\text { accuracy of } 99.3 \% .\end{array}$
\end{tabular}

$\begin{array}{cc}2 & \text { Frequency } \\ & \text { domain } \\ & \text { features, time- } \\ \text { domain } \\ \text { features, non- } \\ \text { linear } \\ \text { dynamics, } \\ \text { Shannon } \\ \text { entropy, } \\ \text { Poincare plot } \\ \text { Age, Systolic } \\ \text { amplitude, } \\ \text { Peak to peak } \\ \text { interval, Pulse } \\ \text { width, } \\ \text { Augmentation } \\ \text { index }\end{array}$
PPG pulse peak power, peak-to-peak intervals

SVM- Cardiac Arrhythmias classification with accuracy of above $90 \%$.

ELM-CVD s risk or healthy with sensitivity of $89.33 \%$, specificity of $90.33 \%$

Artificial Neural Network with linear outputs- premature ventricular contractions

(PVCs) classification with sensitivity of $93.2 \%$, 
specificity of $99.9 \%$

[28]

174 Digital volume pulse (DVP), pulse cycle duration (T), augmentation index (AIx), reflection index (RI) and transit time of reflected wave (RTT)

The acquired signal was displayed and analyzed. First, second and fourth derivatives of the signal were computed. index

measure of Arterial

stiffness
AIx can be better in diagnosis

of CVDs - healthy subject or CVDs

augmentation index - healthy, CVD patients

CVD risk or healthy

SVM- coronary

artery disease (CAD) patients with sensitivity of $85 \%$,

Specificity of $78 \%$. analysis of

PPG signal and its second derivative. and risk assessment strategies: Part I. Circulation, vol. 108 , no. 14 , pp. 1664-72.

[4] Lin W.H., Zhang H and Zhang Y.T. 2013. Investigation on Cardiovascular risk prediction using physiological parameters. Computational and Mathematical Methods in Medicine, vol. 2013, pp. 1-21.

[5] Paradkar N. S. 2017. Non invasive diagnosis of cardiac arrhythmia, coronary artery disease through fingertip Photoplethysmography, Ph. D Thesis, International Institute of Information Technology, Hyderabad.

[6] Elgendi M. 2012. On the analysis of fingertip photoplethysmogram signals. Current Cardiology Reviews, vol. 8, no. 1, pp. 14-25.

[7] Sukor J.A, Redmond S.J and Lovell N.H. 2011. Signal quality measures for pulse oximetry through waveform morphology analysis. Physiological Measurement, vol. 32 no. 3, pp. 369-384.

[8] Gil E, Laguna P, Martinez J, Perez O, Alberola A and Sornmo L. 2013. Heart rate turbulence analysis based on photoplethysmography. Transactions on Biomedical Engineering, vol. 60, no. 11, pp. 3149-3155.

[9] Polanıa L.F, Mestha L. K, Huang D.T and Couderc J.P. 2015. Method for classifying cardiac arrhythmias using photoplethysmography. $37^{\text {th }}$ Annual International Conference on Engineering in Medicine and Biology society, Milan Italy, pp. 6574-7.

[10] Karlen W, Raman, Ansermino J.M, and Dumont G.A. 2013. Multiparameter respiratory rate estimation from the photoplethysmogram. IEEE Transactions on Biomedical Engineering, vol. 60, no. 7, pp. 1946-53.

[11] Jindal V. 2016. Integrating mobile and cloud for PPG signal selection to monitor heart rate during intensive physical exercise. ACM International Conference on Mobile Software Engineering and Systems, New York, USA, pp. 36-37.

[12] Ul-Hoque M.A, Ahsan M.D, Mohajan B, 2011. Measurement of blood pressure using photoplethysmography. In: $13^{\text {th }}$ International Conference on Modeling and Simulation, Cambridge, United Kingdom, pp. 32-35.

[13] Mahri N, Beng G. K, and Ali M. A. M. 2012. Best pulse selection of photoplethysmography signal through comparative method. International Conference on Biomedical Engineering, Penang, Malaysia, pp. 389392.

[14] Yousef Q, Reaz M. B. I, Ali M. A. M. 2012. The analysis of PPG morphology: investigating the effects of aging on arterial compliance. Measurement Science Review, vol. 12 , no. 6 , pp. $266-271$

[15] Agro D, Canicatti R, Tomasino A, et al. 2014. PPG Embedded System For Blood Pressure Monitoring. AEIT Annual Conference - From Research to Industry: The Need for a More Effective Technology Transfer, Trieste, Italy, pp. 1-6.

[16] Lopez-Silva S. M, Dotor M. L, Silveira J. P. 2010. Pulse rate measurement from transmittance photoplethysmography in cycle ergometer test. Instrumentation and Measurement Technology IEEE Conference, Austin, TX, USA, pp. 1039 - 1043.

[3] Naghavi M, Libby P and Falk E. 2003. From vulnerable plaque to vulnerable patient: a call for new definitions 
[17] Lin W.H, Zhang H, and Zhang Y.T. 2013. Investigation on cardiovascular risk prediction using physiological parameters. Computational and Mathematical Methods in Medicine. vol. 2013, Article ID 27269, pp. 1-21.

[18] Radhimeenakshi S. 2016. Classification and prediction of heart disease risk using data mining techniques of Support Vector Machine and Artificial Neural Network. In: 3rd International Conference on Computing for Sustainable Global Development, New Delhi, India, pp. 3107-3111.

[19] Selvaraj N, Jaryal A, Santhoshx J, Deepak K. K and Anand S. 2008. Assessment of heart rate variability derived from finger-tip photoplethysmography as compared to electrocardiography. Journal of Medical Engineering \& Technology, vol. 32, no. 6, pp. 479-84.

[20] Allen J. 2007. Photoplethysmography and its application in clinical physiological measurement. Phsiological Measurement, vol. 23, no. 3, pp. 1-39.

[21] Allen J, Overbeck K, Stansby G and Murray A. 2006. Photoplethysmography assessments in cardiovascular disease, Measurement \& Control, vol. 39, no. 3, pp. 8083.

[22] Shobitha S, Sandhya R, Niranjana Krupa B and Ali M. A. M. 2016. Recognizing cardiovascular risk from photoplethysmogram signals using ELM. $2^{\text {nd }}$ International Conference on Cognitive Computing and Information Processing, Mysore, India, pp. 1-5.

[23] Ukil A, Bandyoapdhyay S, Puri C, Pal A and Mandana K. 2016. Cardiac condition monitoring through photoplethysmogram signal denoising using wearables: Can we detect coronary artery disease with higher performance efficacy? Computing in Cardiology Conference, Vancouver, BC, Canada, pp. 1-4.

[24] Sunil Kumar P, Hari Kumar R. 2015. Performance comparison of EM, MEM, CTM, PCA, ICA, entropy and MI for photoplethysmography Signals. Biomedical \& Pharmacology Journal, vol. 8, no. 1, pp. 413-418.
[25] Solosenko A, Petreenas A, Marozas V. 2015. Photoplethysmography-based method for automatic detection of premature ventricular contractions. Journal of IEEE Transactions on Biomedical Circuits and Systems, vol. 9, no. 5, pp. 662-9.

[26] Foo J. Y and Wilson SJ, 2006. A computational system to optimise noise rejection in photoplethysmography signals during motion or poor perfusion states", International Federation for Medical and Biological Engineering \& Computing, vol. 44, no. 1, pp. 140-5.

[27] Das S, Pal S and Mitra M. 2016. Real time heart rate detection from ppg signal in noisy environment. International Conference on Intelligent Control Power and Instrumentation, Kolkata, India, pp. 70-73.

[28] Rubins U, Grube J, Kukulis I. 2008. Photoplethysmography analysis of artery properties in patients with cardiovascular diseases. In 14th NordicBaltic Conference on Biomedical Engineering and Medical Physics, Riga, Latvia, pp. 319-322.

[29] R. Gonzalez, A. Delgado A, J. Padilla, B. Trenor, J. Ferrero, J. Saiz, Photoplethysmographic augmentation index as a non invasive indicator for vascular assessments, Proceedings of the International Federation for Medical and Biological Engineering Springer 22 (2008) 1167-1170.

[30] Manimegalai P, Delpha J and Thanushkodi K. 2012. An early prediction of cardiac risk using augmentation index developed based on a comparative study, International Journal of Computer Applications vol. 49, no. 15, pp. 27 32.

[31] Paradkar N and Chowdhury S. R. 2017. Coronary artery disease detection using photoplethysmography. 39th Annual International Conference of the IEEE Engineering in Medicine and Biology Society (EMBC), Seogwipo, South Korea, pp. 100-103. 